\title{
Reducing public transit compounds social vulnerabilities during COVID-19
}

\author{
Armita Kar ${ }^{1}$, Andre L. Carrel ${ }^{2,3}$, Harvey J. Miller ${ }^{1,4}$, Huyen T. K. Le ${ }^{1,5,{ }^{*}}$ \\ 1 Department of Geography, The Ohio State University, Columbus, OH 43210 \\ 2 Department of Civil, Environmental and Geodetic Engineering \\ ${ }^{3}$ Knowlton School of Architecture, City and Regional Planning Section, The Ohio State University, \\ Columbus, $\mathrm{OH} 43210$ \\ ${ }^{4}$ Center for Urban and Regional Analysis, The Ohio State University, Columbus, OH 43210, USA \\ ${ }^{5}$ Sustainability Institute, The Ohio State University, Columbus, OH 43210, USA \\ * Corresponding author: Huyen T. K. Le \\ Email: le.253@osu.edu
}

Author Contributions: $\mathrm{AK}$ and HTKL designed research; $\mathrm{AK}$ collected data and conducted analyses; AK, ALC, HJM, and HTKL interpreted the results; AK and HTKL wrote the manuscript; AK, ALC, HJM, and HTKL edited the manuscript; HTKL supervised the project.

Competing Interest Statement: None.

Classification: Social Sciences, Sustainability Science

Keywords: equity, disruption, built environment, urban form, public transportation

This manuscript is currently under review. 


\title{
Public transit cuts during COVID-19 compound social vulnerability
}

\begin{abstract}
The COVID-19 pandemic has severely impacted public transit services through a combination of plummeting ridership during the lockdown and subsequent budget cuts. This study investigates the equity impacts of reductions in accessibility due to public transit service cuts during COVID-19 and their association with urban sprawl. We evaluated accessibility to essential services such as grocery stores and both urgent and non-urgent health care across 22 cities across the United States in three phases during 2020: pre-lockdown, lockdown, and post-lockdown. We estimated the spatio-temporal coverage of transit service during the peak and off-peak periods in each phase. We found stark disparities in food and health care access for various socio-economic groups. Economically disadvantaged and suburban neighborhoods were more likely to lose food and health care access by public transit during COVID-19. In particular, transit service cuts worsened accessibility for population groups with multiple social vulnerabilities, such as low-income workers with zero vehicle ownership, poor households living in urban neighborhoods, and non-white populations residing in suburban neighborhoods. Moreover, our study suggests that sprawled cities experienced greater losses in access to food and health care during COVID-19 than compact cities, highlighting the influence of urban form on the functionality of transit services during crises.
\end{abstract}

\section{Significance Statement}

This study highlights the inequalities and socio-spatial disparities in access to basic services and employment in food and healthcare. Our results thus contribute to understanding the vulnerability and resilience of transit services for diverse social groups and urban forms during shocks and disruptions such as the COVID-19 pandemic. As such, the study implies the value of policies and strategies to rebuild inclusive public transit systems for communities with greater vulnerability. 


\section{Introduction}

For decades, economically and racially marginalized communities in the US have persistently experienced hurdles in accessing basic opportunities such as jobs, schools, health care, food, and other daily activities (1-4). The greater vulnerability of these communities exposed them to disproportionate risks during the COVID-19 pandemic $(5,6)$. Prior studies revealed that marginalized populations are not only at a higher risk of experiencing health-related impacts (e.g., higher infection and death rates) but are also more prone to the socio-economic consequences of the COVID-19 pandemic, such as job losses, food insecurity, and mental stress $(5,7-12)$.

Marginalized populations also depend on infrastructure and services such as public transit that are vulnerable to disruption during pandemics and other shocks. The decline in transit use during COVID-19 was found to be lowest among low-income, less educated, non-white, and female riders (13-15); many were essential workers employed in industries such as food and health that cannot easily accommodate working from home $(16,17)$. As a result, transit service cuts may exacerbate pre-existing social and economic vulnerabilities: on the one hand, service reductions impede workrelated travel, increasing the potential for job losses and financial hardship in marginalized communities, as well as a loss of workforce and productivity for society at large. On the other hand, a lack of access to healthy food and health care can result in nutritional deficiencies, poor health, lack of productivity, and diminished community well-being (18-20). In addition, COVID-19 highlights some major deficiencies in US urban systems and current planning practices $(21,22)$.

Accessibility refers to the ease of reaching destinations (e.g., jobs or services) from key travel origins, such as home (23). Transit accessibility measures the ability to do so using transit services, and therefore, depends on the transit network, schedules, and urban form (24-26). Access to public transit is particularly crucial for marginalized communities to reach and utilize essential services due to their low levels of automobile access. However, urban sprawl, low-density development, and multi-jurisdictional governance in US cities have made the provision of public transit services less cost-effective (27-29). The expansion of highways and continued development outside of urban cores have led to residents, jobs, and services moving to suburbs with more reliance on private vehicles. Moreover, this horizontal expansion of cities resulted in ongoing challenges for transit systems that must contend with declining funding and corresponding needs for cost-cutting to cover an ever-increasing service area (30-32). Historically, sprawling urban development left low-income and vulnerable populations in deteriorating urban neighborhoods (33), but more recently, less affluent populations have been moving more into suburban areas due to gentrification and decreasing affordability of inner urban neighborhoods in many jurisdictions $(34,35)$. As a consequence, the mobility of marginalized communities is further compromised due to limited transportation options and longer travel times, in turn limiting access to jobs, services, recreation, and social interaction (34-36).

The pandemic further negatively affected public transit services of US cities, leaving their survival at stake. Several studies showed that transit use is not a major means of disease transmission if proper sanitization procedures and face mask requirements are in place (37). Yet, transit ridership declined significantly during COVID-19, due to the closure of businesses, teleworking, and fears among transit users of possible transmission, especially those with prior experiences of crowding $(13,14,38,39)$. In response to lower transit demand and financial constraints, transit agencies adopted various measures, such as limiting service coverage and routes, reducing schedules, and modifying fare collection methods $(37,42)$. These changes during COVID-19 have reduced the ability of transit systems across the US to provide adequate services and have raised questions about their ability to recover from the current shock and their resilience to future shocks.

Few studies have investigated the equity outcomes of transit service cuts during COVID-19 (42) and their association with urban form. We aim to address this gap while focusing on access to food and health care. Our objectives are to (1) identify social vulnerabilities induced by transit service 
cuts and the subsequent loss of access to food and health care during COVID-19, and (2) elicit the association between urban sprawl and reduction in accessibility due to transit service cuts. Here, we quantified transit-based access to food and non-urgent and urgent health care within 30 minutes and 45 minutes traveled for all census block groups in the study areas. Our analysis accounts for the peak and off-peak periods due to their difference in frequency of service. To quantify transit service cuts, we considered three phases of pandemic response in 2020, namely, the pre-lockdown (January-February), lockdown (March-June), and post-lockdown phase (November-December).

\section{Results}

\section{Social vulnerabilities induced by transit service cuts}

We found significant declines in transit accessibility to food and health care services for vulnerable populations (Fig.1). Census block groups with a larger share of underprivileged populations (i.e., high poverty rates and large share of low-income workers) were more likely to lose access to food and non-urgent health care facilities due to transit service cuts. Furthermore, suburban block groups were more likely to lose transit access to all these essential services. It is noteworthy that this loss of access affects not only grocery customers and health care clients, but also employees who operated these essential services during the pandemic. A web map of accessibility changes in all cities during the study periods is available in the Supplementary Information.

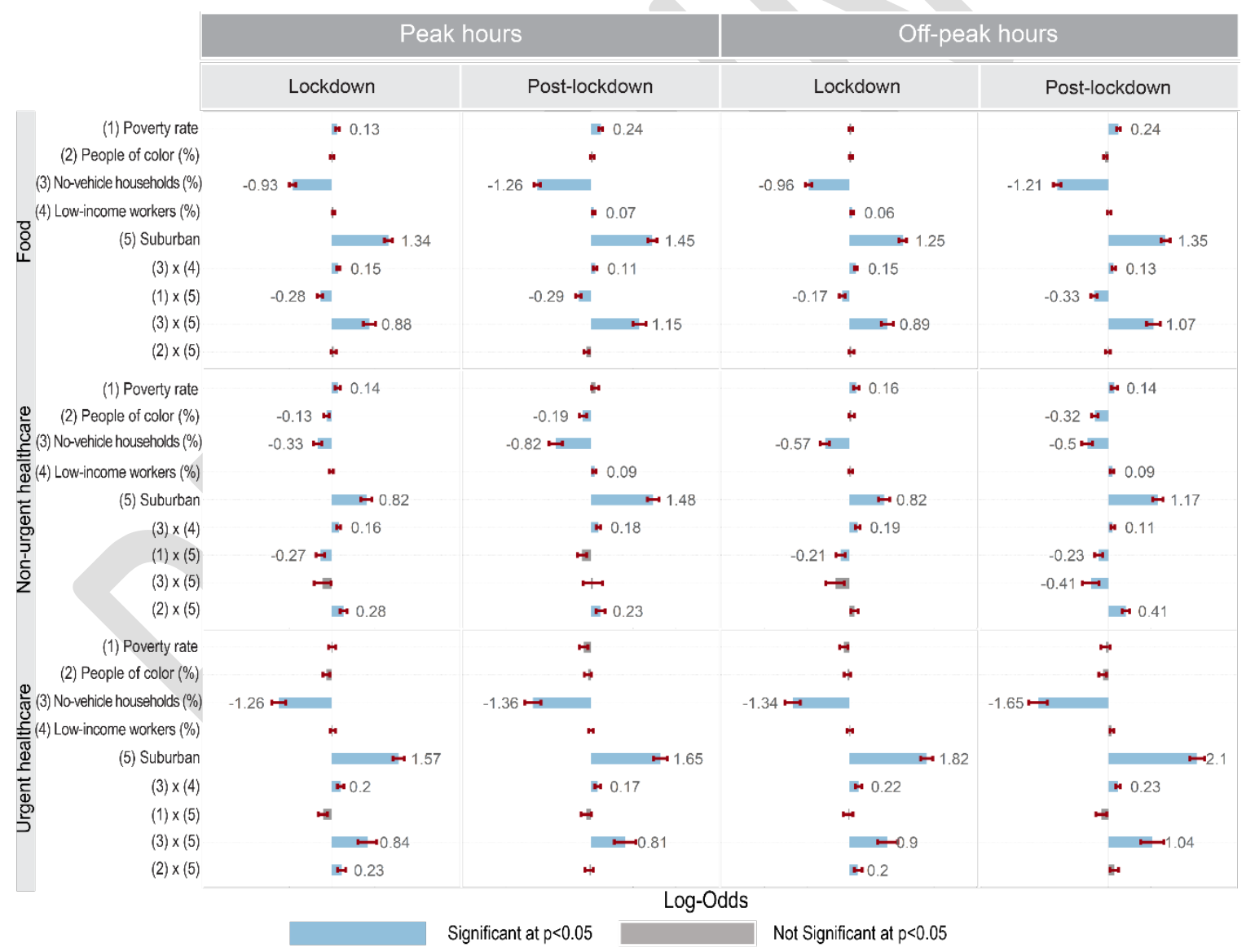

Fig. 1. Likelihood of losing transit access to food and health care during COVID-19. Coefficients estimated from multilevel binary logit models using a travel time threshold of 30 minutes from food and health care facilities. All variables were measured at the census block group level. Blue and grey bars respectively represent the significant and non-significant coefficients of multilevel binary logit models. Coefficients were significant at the $p<0.05$ level. The values of significant coefficients are mentioned beside the blue bars. Red lines represent $95 \%$ confidence intervals. Dependent variable: loss of access (yes/no). Socio-demographic 
and urban form variables were extracted from the American Community Survey and Smart Location Database (see Materials and Methods, and SI for details).

Social vulnerabilities appeared to compound: block groups with multiple social disadvantages experienced a higher likelihood of losing accessibility (Figs. 1 and 2). The likelihood of losing accessibility (shown on the vertical axes in Fig. 2) was higher in block groups with two socioeconomic factors in play, such as income, race, and the level of vehicle ownership. In all study phases, block groups became increasingly more vulnerable to losing access as their shares of both no-vehicle households and low-income workers increase. Similarly, urban areas were more likely to compromise as poverty rate increases, in the case of food and non-urgent health care: urban block groups became more vulnerable to losing transit access if they have a high poverty rate as compared to their suburban counterparts.

While suburban block groups were more likely to lose access, additional disadvantage such as a large share of non-white populations in these block groups further exacerbates their vulnerability in the case of urgent and non-urgent health care (Fig. 2). While a higher share of no-vehicle households associates with continued access in general, suburban block groups with higher novehicle households associate with greater vulnerability of losing access as compared to urban
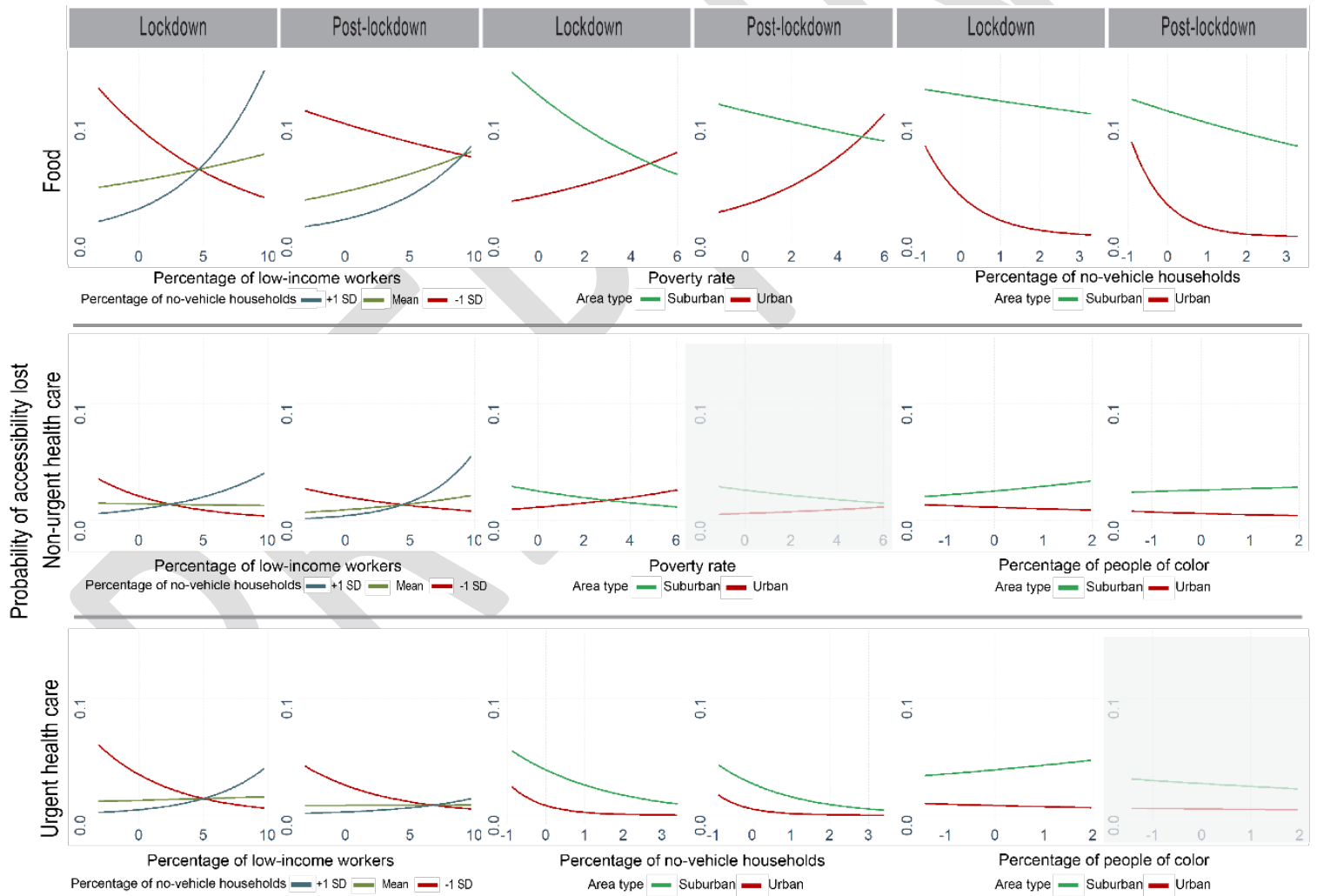

Fig. 2. Interactions between socio-economic variables in predicting the probability of decreased accessibility during peak hours in the lockdown and post-lockdown phases. The vertical axes show the likelihood of reductions in accessibility, given different combinations of socio-economic variables in the $x$ axes. The $x$-axis variables are calculated as the normalized z-score at the census block group level. The interactions were significant at the $p<0.05$ level. Grey box represents insignificant interactions for one out of two phases. Insignificant interactions for both phases are not shown. The interaction patterns of these variables during off-peak hours are similar to those during peak hours (SI Fig. S1) 
areas in the case of food and urgent health care. The direction, magnitude, and interaction patterns of these variables during off-peak hours are similar to those during peak hours, as shown in Fig. 1.

\section{Spatial patterns of reductions in transit-based accessibility}

In the early phase of the pandemic, transit cuts resulted in greater loss of access to food than to health care during the pandemic. In the second phase (post-lockdown), however, most cities showed similar recovery patterns in transit access to both food and health care. The cities that have a greater loss of transit access during lockdown (i.e., have more block groups that lost access) also showed significant recovery of access during the post-lockdown phase (i.e., have fewer block groups that lost access post-lockdown). This recovery pattern was exhibited in Philadelphia, Salt Lake City, Atlanta, Madison, and Ann Arbor. Meanwhile, some cities experienced further losses instead of recovering post-lockdown: while they had fewer block groups that lost access during the lockdown phase, they had more block groups that saw additional losses of access post-lockdown. This pattern can be seen in Austin, Chicago, Los Angeles, and New York. Other cities (i.e., Boston, Dallas, Nashville, Columbus, Louisville, San Francisco, Seattle, and San Jose) had lower yet persistent decreases in transit accessibility throughout all study periods. The remaining cities (i.e., Champaign-Urbana, Denver, Phoenix, Portland, and Miami) showed a varied transit recovery pattern between food and health care access. The differences in transit access changes during peak and off-peak hours are minor (SI Fig. S2).

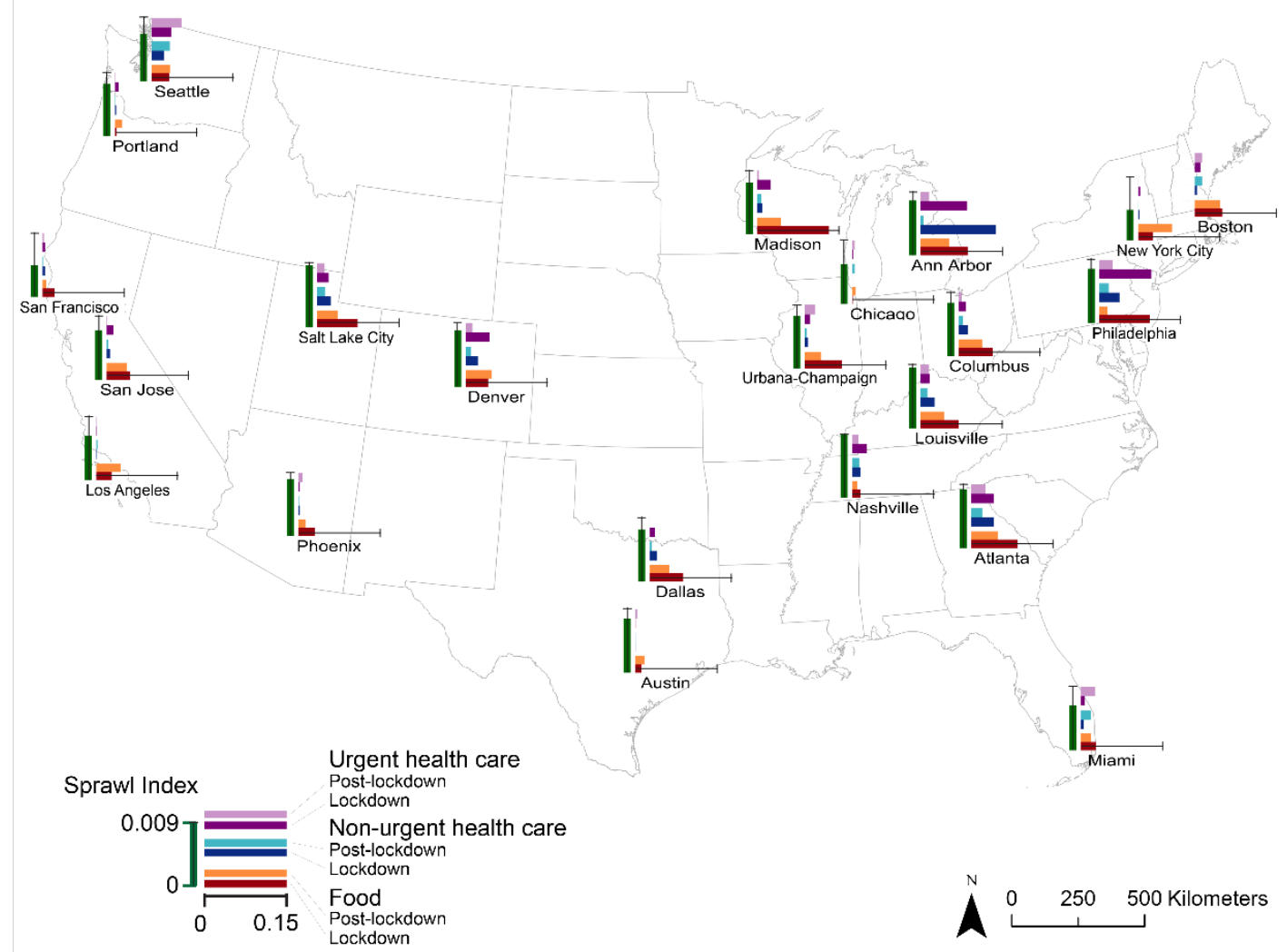

Fig. 3: Probability of losing transit access in lockdown and post-lockdown phases (peak hours only). Horizontal bars on the right represent the probability of losing access to food and healthcare, respectively, estimated from the multilevel binary logit models. The vertical green bar represents the urban sprawl index, with a shorter vertical bar indicates higher compactness. The whiskers on both axes represent the maximum values of urban sprawl and the probability of losing transit access. Boston does not have a vertical bar due to the missing sprawl index. 
Considering that suburban areas were more likely to lose access as suggested by our model results (Fig. 1), we further investigated the association between reduction in transit access and the urban form of these cities. Fig. 4 shows Spearman's rank-order correlation $(\rho)$ between the urban sprawl index and changes in transit access to food and health care (See SI section 6 for more details). We found that the percentage of block groups that lost transit accessibility was positively correlated with the level of sprawl of the cities. This means that both employees and customers of grocery stores and health care facilities in sprawled cities were more likely to lose transit access to these services than those living in compact cities. Additionally, the correlation between the percentage of block groups with access loss and urban sprawl index shows a stronger effect in the lockdown phase than the post-lockdown phase, especially for food and urgent health care.

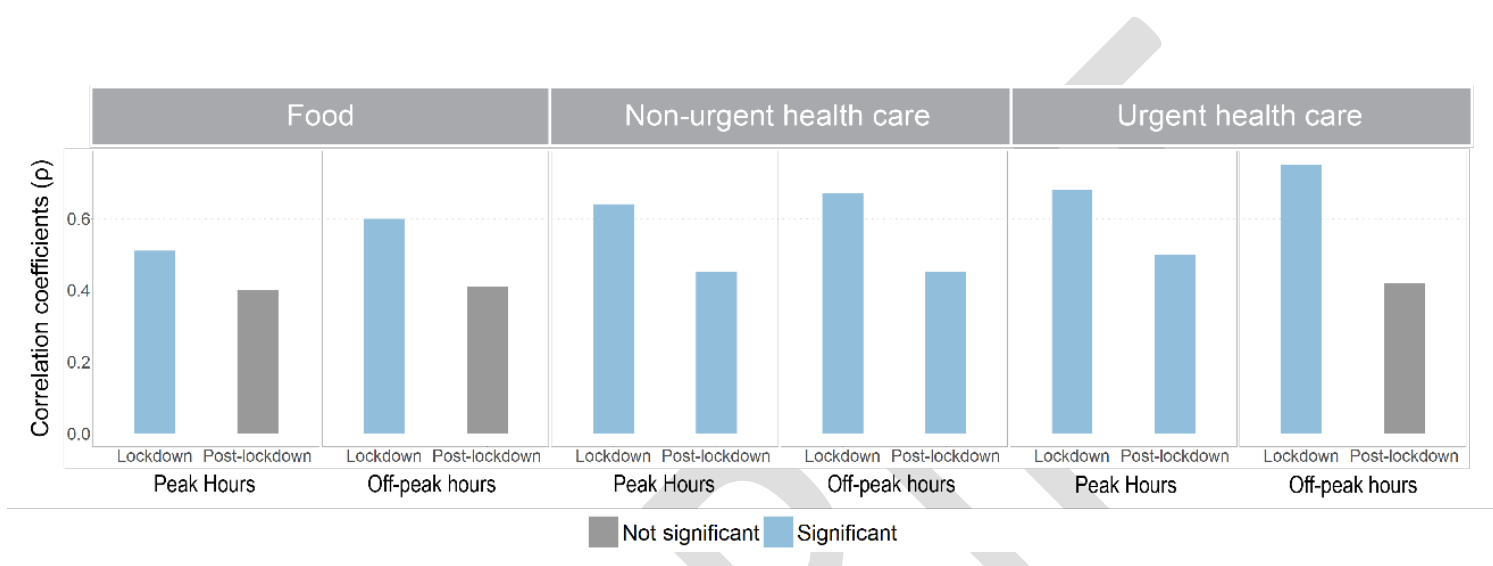

Fig. 4. Correlation between urban sprawl index and the percentage of block groups that lost accessibility in the respective phases. The $y$-axis represents Spearman's rank-order correlation coefficient $(\rho)$. Blue bars represent significant $\rho$ 's (at $p<0.05$ ), and grey bars represent insignificant $\rho$ 's. Higher values of $\rho$ indicate a stronger correlation between urban sprawl and the percentage of block groups with transit service cut. Boston was excluded from this analysis due to the missing sprawl index.

Our robustness checks using a 45-minute travel time threshold show that the results above are robust. The models with 30-minute and 45-minute travel times yielded similar directions of effects for most variables, with few deviations in significance and magnitude (SI Fig. S3). Similarly, our findings on the correlation between urban sprawl and reduction in accessibility remained consistent when the travel time threshold was increased (SI Fig. S4).

\section{Discussion}

\section{Equity implications of accessibility lost during COVID-19}

Access to food and health care is indispensable in everyday life, and they are especially important during a pandemic such as COVID-19. Limited access to such essential services may have particularly adverse effects on disadvantaged populations. For example, limited food access is directly linked to food insecurity and indirectly connected to nutrition deficiencies, higher susceptibility to chronic diseases, and poor physical and mental health (18-20). Similarly, limited health care access is closely related to negative physical and psychological health consequences and may also affect medical care expenses, mortality rates, life expectancy, community well-being, and productivity (43). In addition, grocery stores and health care facilities are basic community services providing employment opportunities for many essential workers $(16,17)$.

Our study highlights inequalities in access to essential services and jobs as a consequence of transit service cuts during to the COVID-19 pandemic, thereby complementing results from recent studies on COVID-19 and social equity (5-7). A considerable portion of both customers and employees of grocery stores and health care facilities are members of marginalized communities 
who rely on public transit for everyday travel (13-17). Our analysis of the equity impacts of reduced access during the COVID-19 pandemic across 22 US cities found that transit services were more severely cut for the communities who needed it the most, particularly low-income populations. Additionally, communities with multiple disadvantages, such as low-income, no vehicle ownership, poor households living in urban areas, and people of color residing in suburban areas, suffered the most from these reductions in accessibility due to transit service cuts. It is particularly important to understand and alleviate these impacts since budget cuts may result in service reductions that outlast the pandemic.

Our study also underscores a critical planning issue in US cities: the compromised resilience and inefficiency in providing crucial community services, including transportation, food, and health care, as a result of urban sprawl. The results indicate greater reductions in food and health care access in sprawled cities and in suburban block groups of all cities. This can be explained by the inefficiency of providing public transit service in these areas: sprawled cities and suburbs often require more physical, financial, and human resources to develop and maintain urban infrastructure and operations (27-29). Therefore, they are more likely to encounter service cuts that are aimed at efficiency during times of crisis and limited resources, such as the COVID-19 pandemic. These findings lend support to the benefits of urban densification, in which the social equity benefits are evident, in addition to the well-established benefits such as reductions in greenhouse gas emissions, energy consumption, and increase in cost-effectiveness of municipal services (44-48). Our study lends support to the existing evidence that a shift from car-oriented, sprawled development to transit-oriented, compact development can promote urban resilience and empower communities (49). In this regard, urban planning practitioners may consider urban densification strategies such as intensifying economic activities, mixed land use development, vertical land (re)development, or the creation of informal urban green spaces. These measures may be adopted at various geographic scales while ensuring the equitable distribution of benefits and minimizing gentrification $(46,49-52)$.

It is worth noting that redesigning the urban system alone may not be sufficient to improve the resilience of public transit services by enabling improved efficiency and better accessibility. Our data suggest that transit agencies who went through larger reductions during the lockdown phase are recovering at a better pace. On the contrary, agencies with lower service reductions in the lockdown phase were mostly forced to maintain those cuts or make additional cuts in the postlockdown phase. It is possible that transit agencies of these cities experienced a slower recovery due to pandemic-induced budget shortages or low ridership. Regardless of the causes, this poor recovery pattern is alarming, as its continuation may put the viability of transit systems at stake. Hence, along with changes in urban structure, the resilience of the transportation system and basic community services also need to be systematically and organizationally strengthened by means of resource allocation, fiscal policies, a skilled workforce, state, and federal support and coordination, and preparedness plans for future shocks.

Our study has significant implications for the understanding of equity issues and the future recovery of public transit, which has historically functioned as a social stabilizer. Reductions in transit services cause reduced access to jobs, basic services, and social connections. If these service cuts persist after the lockdown, the consequences will persist as well. Our findings further illustrate the outsize impacts of COVID-19 on disadvantaged populations and the risk of backsliding on social and economic equity. Greater government assistance for transit agencies in such a time of crisis, and continued updates to planning policies to reduce urban sprawl, are a means to empower affected communities with improved mobility and access to basic services. Given the multiple facets of service cut impacts, as illustrated by our study, multi-agency cooperation and holistic consideration of social and health impacts of transit service adjustments would be beneficial (53). In addition, our study adds to prior evidence regarding the role that urban sprawl and car-oriented development play in driving urban inequality. We recommend that cities use the opportunity presented by the COVID-19 disruption to rethink and redesign urban areas in more resilient ways. 


\section{Conclusions}

Our study investigates the equity impacts of reductions in accessibility to essential services due to transit service cuts during COVID-19. We found compounding effects of social vulnerabilities, such as income, race, lack of vehicle ownership, and neighborhood type, further exacerbating the negative impacts of the loss of transit-based access to food and health care. This study has important implications for designing a systematic method of evaluating temporal changes in public transit services. Future studies may build on this research to evaluate the resilience of other sustainable forms of transportation (e.g., walking and biking) during the COVID-19 pandemic. Future research should also study the transit agencies where slower recovery patterns were observed in order to identify the underlying social, financial, and institutional mechanisms affecting their resilience. In addition, researchers may investigate the various service adjustment strategies employed by agencies to identify approaches with fewer negative equity impacts and apply that knowledge to devise policies for faster recoveries from future disruptions.

Our study has several limitations. First, although we applied an average isochrone measure to account for transit frequency, the service changes we measured with our approach may deviate to a small degree from the actual changes. Second, our analysis of the relationship between urban sprawl and reductions in accessibility is based on a simple correlation measure and does not reveal a causal relationship. The correlations found in this study might be an outcome of external effects that future studies can explore. Finally, although our study intended to select a mix of cities with different sizes, the proportions of big, mid-sized, and small cities are not equal in our selection and are not presentative for all US cities and thus limit the generalizability of our results.

\section{Materials and Methods}

Data source and time frame. We selected 22 cities for this study based on the availability of General Transit Feed Specification (GTFS) data and with the goal of obtaining a mix of cities of different sizes. The cities include Ann Arbor (MI), Atlanta (GA), Austin (TX), Boston (MA), Champaign-Urbana (IL), Chicago (IL), Columbus (OH), Dallas (TX), Denver (CO), Los Angeles (CA), Louisville (KY), Madison (WI), Miami (FL), Nashville (TN), New York City (NY), Philadelphia (PA), Phoenix (AZ), Portland (OR), San Jose (CA), Seattle (WA), San Francisco (CA), and Salt Lake City (UT). In each city, we included all transit modes (i.e., bus, subway, light rail, and cable tram) for which data were available. We obtained static GTFS data with information about transit routes, stops or stations, schedules, and transfers from the OpenMobilityData website (54), and road network data from OpenStreetMap (OSM) (55).

Based on the changes in transit services in 2020, we determined three phases of pandemic response, namely pre-lockdown, lockdown, and post-lockdown, to examine the temporal variation of transit service coverage. The pre-lockdown phase extended from January to February as states began issuing official stay-at-home orders in March 2020 (56). We designated March to June as the lockdown phase and November to December as the post-lockdown phase since the study areas experienced the largest declines in transit services and subsequent recoveries (if any) within these time windows. We selected a Tuesday within each phase to measure accessibility provided by transit on a regular weekday (all weekdays followed the same transit schedule). We chose the specific dates for each transit agency separately, using the following criteria: (1) pre-lockdown period: a date on which the highest number of transit routes were served, (2) lockdown period: a date on which the lowest number of transit routes were served, and (3) post-lockdown period: a date on which the highest number of transit routes was served. If multiple Tuesdays fulfilled the criteria, we used the first such Tuesday to perform our analysis. SI Table S1 provides the full information of transit agencies and dates used in this study. 
In this study, access to food represents peoples' ability to reach grocery stores that carry a wide assortment of fresh and healthy food products. We obtained the locations and business data of grocery stores for each city from InfoGroup (57). We filtered grocery stores based on the North American Industry Classification System (NAICS) code, retailer brand names, and scale of businesses. The grocery stores used in this study represent the top 10th percentile of supermarkets and grocery stores based on the employee size and sales volume of each store across 22 cities (SI Table S2). We also included warehouse clubs, supercenters, and department stores that sell grocery products, namely, Walmart, Target, Costco Wholesale, Sam's Club, and BJ's.

We collected health care locations from InfoGroup as well. We classified the locations into urgent (general hospital and emergency care services) and non-urgent health care facilities (specialty hospitals and primary care services) based on their NAICS code and (SI Table S3). Our study areas contain many primary care and emergency care facilities that are spatially clustered. Hence, we applied the DBSCAN clustering approach $(58,59)$ on these two datasets to cluster the data and thus reduce the computational burden. The detailed food and health care location selection criteria are discussed in SI Section 2.

Measuring accessibility to food and health care. We analyzed changes in transit-based accessibility to food, urgent and non-urgent health care across the 22 cities. We generated a halfmile buffer around each transit stop (approximately 10 minutes walking distance), based on local transit network during the pre-lockdown period. We defined the following criteria to generate isochrones of travel time (i.e., accessibility): (1) travelers only use transit and walking, and walk no more than half a mile to and from transit stops, and (2) travelers transfer no more than once, and each transfer lasts no more than 5 minutes.

For each city, we generated 30-minute and 45-minute isochrones around each grocery store, urgent or non-urgent health care facility for peak and off-peak hours of each study phase. We used $9 \mathrm{AM}-10 \mathrm{AM}$ as an example of the peak hours and $1 \mathrm{PM}-2 \mathrm{PM}$ as an example of the off-peak hours and sampled isochrones for both hours using 10-minute intervals. Finally, we represented accessibility as a geometric average of the sample isochrones for peak or off-peak hours for each destination to account for variation in waiting time. The process of generating and processing Isochrones is discussed in SI Section 3.

Quantifying equity impacts of public transit service changes. We extracted socio-economic information at the census block group level from American Community Survey (ACS) 5-year estimates (2014-2018) and the Environmental Protection Agency's Smart Location Database (60, 61) (SI section 4). We used separate multilevel binary logit models to estimate the equity impacts of reduced accessibility during peak and off-peak hours in the lockdown and post-lockdowns phases as compared to the pre-lockdown phase. Our dependent variable is a binary variable denoting whether or not a block group lost transit access during the specified time period of each model. Level 1 includes block-group-level socioeconomic factors, whereas level 2 includes cities as random intercepts. SI Section 5 describes more details about the multilevel binary logit models.

Evaluating the relationship between urban sprawl and accessibility reduction. We acquired the urban compactness dataset from the National Cancer Institute (62) and derived the urban sprawl index as an inverse of the area-weighted average of this county-level compactness data. A lower sprawl index indicates more compact whereas a higher sprawl index indicates more sprawl. SI Section 6 provides further details on estimating the sprawl index for each city.

We estimated the percentage of block groups of each study area that experienced a reduction in accessibility during the peak and off-peak periods of the lockdown and post-lockdown phases compared to the pre-lockdown phase using travel time thresholds of 30 and 45 minutes. Finally, 
we tested the association between the urban sprawl index and the percentage of block groups with reduced accessibility for our study areas using Spearman's rank-order correlation test.

Data and code availability

All code are published here: https://github.com/armitakar/Transit-based-Access-COVID-19

\section{References}

1. M. B. Elmes, Economic Inequality, Food Insecurity, and the Erosion of Equality of Capabilities in the United States. Business \& Society 57, 1045-1074 (2018).

2. H. Allcott, et al., Food Deserts and the Causes of Nutritional Inequality*. The Quarterly Journal of Economics 134, 1793-1844 (2019).

3. R. Yearby, Racial Disparities in Health Status and Access to Healthcare: The Continuation of Inequality in the United States Due to Structural Racism. American Journal of Economics and Sociology 77, 1113-1152 (2018).

4. T. Mouw, A. L. Kalleberg, Occupations and the Structure of Wage Inequality in the United States, 1980s to 2000s. Am Sociol Rev 75, 402-431 (2010).

5. L. Bowleg, We're Not All in This Together: On COVID-19, Intersectionality, and Structural Inequality. Am J Public Health 110, 917-917 (2020).

6. J. A. Patel, et al., Poverty, inequality and COVID-19: the forgotten vulnerable. Public Health 183, 110-111 (2020).

7. A. van Dorn, R. E. Cooney, M. L. Sabin, COVID-19 exacerbating inequalities in the US. The Lancet 395, 1243-1244 (2020).

8. V. Abedi, et al., Racial, Economic, and Health Inequality and COVID-19 Infection in the United States. J. Racial and Ethnic Health Disparities (2020) https:/doi.org/10.1007/s40615020-00833-4 (April 28, 2021).

9. J. McLaren, "Racial Disparity in COVID-19 Deaths: Seeking Economic Roots with Census data." (National Bureau of Economic Research, 2020).

10. R. Saenz, C. Sparks, "The Inequities of Job Loss and Recovery Amid the COVID-19 Pandemic" (2020) https:/doi.org/10.34051/p/2021.3 (July 30, 2021).

11. L. Montenovo, et al., "Determinants of Disparities in Covid-19 Job Losses" (National Bureau of Economic Research, 2020).

12. A. Kar, Y. Motoyama, A. L. Carrel, H. J. Miller, H. T. K. Le, COVID-19 exacerbates unequal food access. Applied Geography 134, 102517 (2021).

13. L. Liu, H. J. Miller, J. Scheff, The impacts of COVID-19 pandemic on public transit demand in the United States. PLOS ONE 15, e0242476 (2020). 
14. S. Hu, P. Chen, Who left riding transit? Examining socioeconomic disparities in the impact of COVID-19 on ridership. Transportation Research Part D: Transport and Environment 90, 102654 (2021).

15. R. Brough, M. Freedman, D. C. Phillips, "Understanding Socioeconomic Disparities in Travel Behavior during the COVID-19 Pandemic" (UCI Center for Population, Inequality, and Policy, 2020).

16. The Lancet, The plight of essential workers during the COVID-19 pandemic. Lancet 395, 1587 (2020).

17. C. A. Parks, N. B. Nugent, S. E. Fleischhacker, A. L. Yaroch, Food System Workers are the Unexpected but Under Protected COVID Heroes. The Journal of Nutrition 150, 2006-2008 (2020).

18. C. Gundersen, J. P. Ziliak, Food Insecurity And Health Outcomes. Health Affairs 34, 18301839 (2015).

19. C. B. Barrett, Measuring Food Insecurity. Science 327, 825-828 (2010).

20. D. Decker, M. Flynn, Food Insecurity and Chronic Disease: Addressing Food Access as a Healthcare Issue. Rhode Island Medical Journal 101, 28-30 (2018).

21. S. Hamidi, S. Sabouri, R. Ewing, Does Density Aggravate the COVID-19 Pandemic?: Early Findings and Lessons for Planners. Journal of the American Planning Association 86, 495509 (2020).

22. M. Acuto, COVID-19: Lessons for an Urban(izing) World. One Earth 2, 317-319 (2020).

23. D. M. Levinson, H. Wu, Towards a general theory of access. JTLU 13, 129-158 (2020).

24. A. T. Murray, X. Wu, Accessibility tradeoffs in public transit planning. Journal of Geographical Systems 5, 93-107 (2003).

25. D. O'Sullivan, A. Morrison, J. Shearer, Using desktop GIS for the investigation of accessibility by public transport: an isochrone approach. International Journal of Geographical Information Science 14, 85-104 (2000).

26. T. L. Lei, R. L. Church, Mapping transit-based access: integrating GIS, routes and schedules. International Journal of Geographical Information Science 24, 283-304 (2010).

27. J. I. Carruthers, G. F. Ulfarsson, Urban Sprawl and the Cost of Public Services. Environ Plann B Plann Des 30, 503-522 (2003).

28. R. W. Burchell, S. Mukherji, Conventional Development Versus Managed Growth: The Costs of Sprawl. Am J Public Health 93, 1534-1540 (2003).

29. E. Gielen, G. Riutort-Mayol, J. L. Miralles i Garcia, J. S. Palencia Jiménez, Cost assessment of urban sprawl on municipal services using hierarchical regression. Environment and Planning B: Urban Analytics and City Science 48, 280-297 (2021). 
30. A. Richiedei, M. Tira, Municipal Budget Management and the Generation of Urban Sprawl. A Case Study of the Lombardy Region (Italy). Planning Practice \& Research 35, 169-184 (2020).

31. J. C. Sakowicz, Urban Sprawl: Florida's and Maryland's Approaches. J. Land Use \& Envtl. L. 19, 377 (2003).

32. M. Hortas-Rico, Urban sprawl and municipal budgets in Spain: A dynamic panel data analysis. Papers in Regional Science 93, 843-864 (2014).

33. R. Ewing, R. Pendall, D. Chen, Measuring Sprawl and Its Transportation Impacts. Transportation Research Record 1831, 175-183 (2003).

34. J. Allen, S. Farber, "Suburbanization of transport poverty" (SocArXiv, 2020) https:/doi.org/10.31235/osf.io/hkpfj (November 5, 2020).

35. D. Schleith, M. Widener, C. Kim, An examination of the jobs-housing balance of different categories of workers across 26 metropolitan regions. Journal of Transport Geography 57, $145-160$ (2016).

36. Q. Wang, N. E. Phillips, M. L. Small, R. J. Sampson, Urban mobility and neighborhood isolation in America's 50 largest cities. Proc Natl Acad Sci USA 115, 7735-7740 (2018).

37. A. Tirachini, O. Cats, COVID-19 and Public Transportation: Current Assessment, Prospects, and Research Needs. JPT 22 (2020).

38. S.-H. Cho, H.-C. Park, Exploring the Behaviour Change of Crowding Impedance on Public Transit due to COVID-19 Pandemic: Before and After Comparison. Transportation Letters, $1-8(2021)$.

39. S. Ahangari, C. Chavis, M. Jeihani, "PUBLIC TRANSIT RIDERSHIP ANALYSIS DURING THE COVID-19 PANDEMIC" (Public and Global Health, 2020) https:/doi.org/10.1101/2020.10.25.20219105 (April 28, 2021).

40. F. Carozzi, S. Provenzano, S. Roth, Urban Density and COVID-19. 30 (2020).

41. United Nations, "Policy Brief: COVID-19 in an Urban World" (2020).

42. J. DeWeese, et al., A Tale of 40 Cities: A Preliminary Analysis of Equity Impacts of COVID19 Service Adjustments across North America. Findings (2020) https:/doi.org/10.32866/001c.13395 (May 25, 2021).

43. E. H. Bradley, B. R. Elkins, J. Herrin, B. Elbel, Health and social services expenditures: associations with health outcomes. BMJ Quality \& Safety 20, 826-831 (2011).

44. R. Ewing, S. Hamidi, Compactness versus Sprawl: A Review of Recent Evidence from the United States. Journal of Planning Literature 30, 413-432 (2015).

45. S. B. Kashem, A. Irawan, B. Wilson, Evaluating the dynamic impacts of urban form on transportation and environmental outcomes in US cities. Int. J. Environ. Sci. Technol. 11, 2233-2244 (2014). 
46. G. M. Ahlfeldt, E. Pietrostefani, A. Schumann, T. Matsumoto, Demystifying compact urban growth: Evidence from 300 studies from across the world in (2018) https:/doi.org/10.1787/BBEA8B78-EN.

47. B. Stone, A. C. Mednick, T. Holloway, S. N. Spak, Is Compact Growth Good for Air Quality? Journal of the American Planning Association 73, 404-418 (2007).

48. S. Hankey, J. D. Marshall, Urban Form, Air Pollution, and Health. Curr Envir Health Rpt 4, 491-503 (2017).

49. Institute for Transportation and Development Policy (ITDP), Preparing Cities for the Future: An Interview with MOBILIZE Keynote Speaker, Gil Peñalosa. Institute for Transportation and Development Policy (2019) (May 6, 2021).

50. M. Artmann, Urban sprawl, compact urban development and green cities. How much do we know, how much do we agree? Ecological Indicators, 7 (2019).

51. C. D. D. Rupprecht, J. A. Byrne, Informal urban green space as anti-gentrification strategy? 20.

52. Z. Lin, Vertical Urbanism: Re-conceptualizing the Compact City in Cross Americas: Probing Disglobal Networks-Papers, (ACSA Press, 2016), pp. 122-127.

53. SPUR, "Seamless Transit: How to make Bay Area public transit function like one rational, easy-to-use system" (SPUR, 2015) (August 23, 2021).

54. GTFS, OpenMobilityData - Public transit feeds from around the world (2020) (May 3, 2021).

55. OpenStreetMap, OpenStreetMap. OpenStreetMap (2020) (June 17, 2021).

56. A. Moreland, et al., Timing of State and Territorial COVID-19 Stay-at-Home Orders and Changes in Population Movement - United States, March 1-May 31, 2020. MMWR Morb. Mortal. Wkly. Rep. 69, 1198-1203 (2020).

57. InfoGroup, InfoGroup. Infogroup (2019).

58. M. Hahsler, M. Piekenbrock, D. Doran, dbscan: Fast Density-Based Clustering with R. J. Stat. Soft. 91 (2019).

59. R. J. G. B. Campello, D. Moulavi, J. Sander, "Density-Based Clustering Based on Hierarchical Density Estimates" in Advances in Knowledge Discovery and Data Mining, Lecture Notes in Computer Science., J. Pei, V. S. Tseng, L. Cao, H. Motoda, G. Xu, Eds. (Springer Berlin Heidelberg, 2013), pp. 160-172.

60. K. Ramsey, A. Bell, Smart location database. Washington, DC (2014).

61. US Census Bureau, 2014-2018 American Community Survey 5-Year Estimates (2020) (July $31,2020)$.

62. R. Ewing, S. Hamidi, "Measuring urban sprawl and validating sprawl measures" (National Cancer Institute, National Institutes of Health, 2014). 